\title{
Spectral analysis of cool white dwarfs accreting from planetary systems: from the UV to the optical
}

\author{
M. A. Hollands, ${ }^{1,2 \star ~ P .-E . ~ T r e m b l a y, ~}{ }^{2}$ B. T. Gänsicke, ${ }^{2}$ and D. Koester ${ }^{3}$ \\ ${ }^{1}$ Department of Physics and Astronomy, University of Sheffield, Sheffield, S3 7RH, UK \\ ${ }^{2}$ Department of Physics, University of Warwick, Coventry CV4 7AL, UK \\ ${ }^{3}$ Institut für Theoretische Physik und Astrophysik, University of Kiel, 24098 Kiel, Germany
}

Accepted 2021 December 16. Received 2021 December 16; in original form 2021 July 22

\begin{abstract}
The accretion of planetary debris into the atmospheres of white dwarfs leads to the presence of metal lines in their spectra. Cool metal-rich white dwarfs, which left the main-sequence many Gyr ago, allow the study of the remnants of the oldest planetary systems. Despite their low effective temperatures $\left(T_{\text {eff }}\right)$, a non-neglible amount of their flux is emitted in the near ultraviolet (NUV), where many overlapping metal lines can potentially be detected. We have observed three metal-rich cool white dwarfs with the Space Telescope Imaging Spectrograph (STIS) onboard the Hubble Space Telescope (HST), and compare the results determined from the NUV data with those previously derived from the analysis of optical spectroscopy. For two of the white dwarfs, SDSS J1038-0036 and SDSS J1535+1247, we find reasonable agreement with our previous analysis and the new combined fit of optical and NUV data. For the third object, SDSS J0956+5912, including the STIS data leads to a ten percent lower $T_{\text {eff }}$, though we do not identify a convincing explanation for this discrepancy. The unusual abundances found for SDSS J0956+5912 suggest that the accreted parent-body was composed largely of water ice and magnesium silicates, and with a mass of up to $\simeq 2 \times 10^{25} \mathrm{~g}$. Furthermore SDSS J0956+5912 shows likely traces of atomic carbon in the NUV. While molecular carbon is not observed in the optical, we demonstrate that the large quantity of metals accreted by SDSS J0956+5912 can suppress the $\mathrm{C}_{2}$ molecular bands, indicating that planetary accretion can convert DQ stars into DZs (and not DQZs/DZQs).
\end{abstract}

Key words: (stars:) white dwarfs - stars: atmospheres - ultraviolet: stars - planets and satellites: composition

\section{INTRODUCTION}

The majority of exoplanets orbit stars with masses $\leq 4 \mathrm{M}_{\odot}$ which are destined to become white dwarfs, with a large fraction of their planetary systems surviving stellar evolution (Mustill et al. 2014; Veras et al. 2016; Villaver \& Livio 2007). Within the Solar System, planetary bodies beyond 1 au are expected to survive this process (Schröder \& Connon Smith 2008), i.e. Mars, the gas giants, and the asteroid/Kuiper belts will one day orbit a degenerate Sun (Sackmann et al. 1993; Duncan \& Lissauer 1998).

Observationally, remnant planetary systems in orbit of white dwarfs are well established. The intense surface gravities of white dwarfs (typically $\log g \simeq 8$, with $g$ in units of $\mathrm{cm} \mathrm{s}^{-2}$ ), are expected to result in atmospheres devoid of elements heavier than hydrogen or helium, due to rapid gravitational settling (Schatzman 1949; Paquette et al. 1986). The presence of metal lines observed in the spectra of at least one quarter of white dwarfs (Zuckerman et al. 2003, 2010; Koester et al. 2014) indicates that accretion of metal-rich material occurs frequently. Furthermore, circumstellar dust discs are observed around some metal-rich white dwarfs due to excess infra red flux in their spectral energy distributions (Zuckerman \& Becklin 1987; Graham et al. 1990). This led to the hypothesis that exoplanetesimals

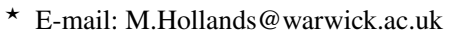

(e.g. asteroids and minor planets) could be perturbed onto orbits with pericentres within the white dwarf Roche radius, leading to their tidal break up, forming a circumstellar debris disc which is then accreted onto the white dwarf surface (Jura 2003). Dynamical simulations of remnant planetary systems indicate that planetesimals can be directed towards the central white dwarf through gravitational encounters with other planetary bodies (Debes \& Sigurdsson 2002; Mustill et al. 2014; Veras \& Gänsicke 2015). Additional observational evidence comes from the presence of gas discs within the circumstellar dust discs (Gänsicke et al. 2006; Farihi et al. 2012; Melis et al. 2012; Gentile Fusillo et al. 2021), which may be related to the presence of intact solid planetesimals embedded within some of the dusty debris discs (Manser et al. 2019). The fundamental hypothesis of planetesimals reaching the tidal disruption radius (Jura 2003) has unambiguously been corroborated by the detection of two systems exhibiting deep and rapidly evolving photometric transits caused by disintegrating planetary bodies (Vanderburg et al. 2015; Vanderbosch et al. 2019; Guidry et al. 2021; Vanderbosch et al. 2021). More recently this body of evidence has come to include bona fide planets orbiting close to the white dwarf - in one case detection of an evaporating giant planet (Gänsicke et al. 2019) and another from photometric transits of a planet crossing the line-of-sight of the white dwarf (Vanderburg et al. 2020).

Among the various observational signatures flagging the presence 
of evolved planetary systems, the most common one is the presence of metal lines in white dwarf spectra. Atmospheric modelling of these spectra, not only establishes the stellar properties, but also the chemical composition of the accreted parent bodies (Zuckerman et al. 2007). This has led to the characterisation of many different types of planetary compositions (Swan et al. 2019), for example, lithospheric material rich in $\mathrm{Ca}$ and $\mathrm{Al}$ (Zuckerman et al. 2011), core-like material rich in Fe and Ni (Gänsicke et al. 2012; Wilson et al. 2015; Hollands et al. 2018), and in one instance (Xu et al. 2017) cometary material rich in volatile elements such as $\mathrm{N}$ and $\mathrm{S}$. Some rocky material has also been established as enriched in water-ice due to the over abundance of oxygen (Farihi et al. 2013; Raddi et al. 2015), and more generally from the presence of trace hydrogen in heliumdominated atmospheres (Gentile Fusillo et al. 2017; Hoskin et al. 2020; Izquierdo et al. 2021). Most recently, atmospheres enriched in the alkali metals, $\mathrm{Li}$ and $\mathrm{K}$, have been identified in a handful of cool white consistent with either accretion of primordial Li-enhancement (Kaiser et al. 2020) or continental-crust like material (Hollands et al. 2021). Additionally, Be has been detected at two systems as a signature of spallation by high energy particles (Klein et al. 2021).

In this work we focus on a specific class of metal-polluted white dwarf, the DZs, which are distinguished by only having metal lines in their spectra. For $T_{\text {eff }}$ below $\approx 10000 \mathrm{~K}$, the strength of helium lines becomes negligible, and so most DZs have helium dominated atmospheres, though below $\approx 5000 \mathrm{~K}$ the hydrogen Balmer series also becomes negligible in strength, and so very cool DZs can also have hydrogen dominated atmospheres. DZs have deep outer convection zones, which keep heavy elements suspended for much longer than compared with the radiative atmospheres of warm DAZs. While the corresponding diffusion timescales are on the order of Myrs, these are still much shorter than the 1-10 Gyr white dwarf cooling ages, thus maintaining the requirement of accretion as the origin of the metals. On the other hand, the Myr diffusion timescales are likely longer than the dusty debris disc lifetime (Girven et al. 2012; Cunningham et al. 2021), and so DZs are rarely observed with the infrared excesses characteristic of debris discs. Because of the low opacity of helium in the outer layers of cool DZs, their atmospheres reach fluid-like densities at the deepest layers relevant to atmospheric modelling (Blouin et al. 2018). This has the effect of making DZ atmospheres challenging to model, as well as leading to extreme pressure-broadening of metal lines formed at these high densities (Allard et al. 2016a,b; Hollands et al. 2017; Blouin et al. 2018, 2019a,b).

Crucial to obtaining correct atmospheric parameters of DZs is an accurate measurement of $T_{\text {eff }}$, as this quantity can be highly correlated with the metal abundances. While most spectroscopic observations of DZ stars are performed at optical wavelengths, for some DZs (particularly the warmest), a significant amount of flux may reside in the near-ultraviolet (NUV). Incorrect determination of the flux level in the NUV, can therefore have a large impact on the determined $T_{\text {eff }}$, and thus impact abundance measurements (Wolff et al. 2002). To establish whether DZ atmospheric parameters can be determined from optical data alone we have acquired NUV spectra of three DZs ${ }^{1}$ whose optical spectra from the Sloan Digital Sky Survey (SDSS) have previously been analysed (Hollands et al. 2017): SDSS J095645.12+591240.7， SDSS J103809.09-003622.2, and SDSS J153505.81+124745.2 (shortened versions used hereafter) using the Space Telescope Imaging Spectrograph (STIS) onboard the Hubble Space Telescope (HST).

1 Strictly speaking, SDSS J0956+5912 is classed as a DZA due to the pres-

\section{OBSERVATIONS}

All three stars, SDSS J0956+5912, SDSS J1038-0036, and SDSS J1535+1247, have ample photometry spanning the nearultraviolet to the near-infrared. catalogues. Furthermore, as of Gaia eDR3, all three objects have 5-parameter astrometric solutions. The available astrometric and photometric data for the three stars is given in Table 1.

\subsection{Optical spectra}

For SDSS J1038-0036 and SDSS J1535+1247, in 2013 we obtained spectra covering 3100-10000 $\mathrm{A}$ using the Intermediate-dispersion Spectrograph and Imaging System (ISIS) on the William Herschel telescope (WHT). Details of the instrumental setup and basic calibration have been summarised by Hollands et al. (2017). However we apply an improved flux calibration to these spectra in order to increase the accuracy of our spectroscopic fitting. The approach used is similar to the method for correcting the flux calibration of SDSS BOSS spectra which we described in Hollands et al. (2017). For the ISIS blue arm, we fit the differences between the SDSS $u$ and $g$ magnitudes and the synthetic magnitudes of the WHT spectra. This provided a wavelength dependent correction to the flux calibration. Similarly for the ISIS red arm, we calculate synthetic magnitudes for the $r, i$, and $z$ filters, and again fit the differences from the SDSS photometry with a straight line to correct the flux calibration.

For SDSS J0956+5912 we have obtained additional spectra using the OSIRIS spectrograph on the GTC in service mode as part of ITP programme GTC1-16ITP. Four $u$-band spectra using the R2500U grating were taken on the 4th January 2017, each with 900 s exposure times. Additionally four $i$-band spectra were taken using the R2500I grating on the 5th February 2017, again using 900 s exposures. Standard GTC service-mode calibration observations were also taken, i.e. bias frames flat fields, arcs, and flux standards. Extraction of the 1D spectra was performed using the starlink suite of software, with wavelength and flux calibrations performed using molly ${ }^{2}$.

\subsection{HST data}

The three white dwarfs SDSS J0956+5912, SDSS J1038-0036, and SDSS J1535+1247, were observed using HST STIS, using the G230L grating and the two arcsec wide slit from April to August 2016. This setup covered wavelengths from $1580-3140 \AA$ with a resolving power of $R \simeq 500$. For SDSS J1038-0036 and SDSS J1535+1247, these wavelengths effectively cover their full SEDs when combined with our WHT spectra which go as blue as $3100 \AA$. For SDSS J0956+5912, there remains a gap in observations between the HST and GTC spectra, though we found that all elements expected to be observed in that range already had at least some lines at other wavelengths covered by our data. Standard wavelength and flux calibrations were used throughout. The observations were performed in time-tag mode, though we did not detect any signature of variability in our data. Perorbit spectra were coadded for the spectra used for fitting, however for SDSS J1535+1247, guidance was lost during the final $240 \mathrm{~s}$ of the orbit, as evidenced by the time-tagged lightcurve. Therefore for the final co-add, the fluxes of this sub-spectrum were scaled accordingly with the amount of time lost. A summary of observations is provided

ence of $\mathrm{H} \alpha$ and $\mathrm{H} \beta$ in its spectrum. However, for brevity we consider this object as a DZ when collectively referring to all three stars.

2 molly software can be found at https://deneb.astro.warwick.ac. uk/phsaap/software/ 
Table 1. Astrometry and photometry for the white dwarfs observed with HST STIS. For GALEX NUV photometry, the first number is the value obtained by GALEX, whereas the second number was calculated by integrating our STIS spectra over the GALEX bandpass.

\begin{tabular}{lccc}
\hline & SDSS J0956+5912 & SDSS J1038-0036 & SDSS J1535+1247 \\
\hline Ra & $09: 56: 45.147$ & $10: 38: 09.091$ & $15: 35: 05.810$ \\
Dec & $+59: 12: 44.64$ & $-00: 36: 22.15$ & $+12: 47: 45.20$ \\
$\mu_{\text {Ra }}\left[\right.$ mas yr $^{-1}$ ] & $-110.588 \pm 0.088$ & $-92.833 \pm 0.096$ & $-165.597 \pm 0.034$ \\
$\mu_{\text {Dec }}\left[\right.$ mas yr $^{-1}$ ] & $-31.488 \pm 0.131$ & $19.157 \pm 0.112$ & $-179.697 \pm 0.033$ \\
parallax [mas] & $7.4116 \pm 0.1219$ & $16.8686 \pm 0.0895$ & $51.9461 \pm 0.0344$ \\
\hline GALEX NUV & $21.42 \pm 0.27 / 21.03 \pm 0.01$ & $19.67 \pm 0.08 / 19.74 \pm 0.01$ & $22.41 \pm 0.39 / 22.28 \pm 0.03$ \\
SDSS $u$ & $18.950 \pm 0.021$ & $17.722 \pm 0.010$ & $18.039 \pm 0.013$ \\
SDSS $g$ & $18.378 \pm 0.007$ & $16.993 \pm 0.004$ & $15.978 \pm 0.003$ \\
SDSS $r$ & $18.403 \pm 0.009$ & $16.948 \pm 0.005$ & $15.469 \pm 0.003$ \\
SDSS $i$ & $18.547 \pm 0.011$ & $17.079 \pm 0.005$ & $15.430 \pm 0.003$ \\
SDSS $z$ & $18.747 \pm 0.035$ & $17.308 \pm 0.013$ & $15.497 \pm 0.005$ \\
PanSTARRS $g$ & $18.3948 \pm 0.0067$ & $16.9718 \pm 0.0084$ & $15.9270 \pm 0.0057$ \\
PanSTARRS $r$ & $18.4147 \pm 0.0057$ & $16.9568 \pm 0.0020$ & $15.5091 \pm 0.0005$ \\
PanSTARRS $i$ & $18.5713 \pm 0.0046$ & $17.1095 \pm 0.0038$ & $15.4663 \pm 0.0042$ \\
PanSTARRS $z$ & $18.7814 \pm 0.0149$ & $17.3335 \pm 0.0041$ & $15.5434 \pm 0.0070$ \\
PanSTARRS $y$ & $18.8534 \pm 0.0216$ & $16.9401 \pm 0.0133$ & $15.5532 \pm 0.0072$ \\
Gaia $G$ & $18.3839 \pm 0.0014$ & $16.9480 \pm 0.0012$ & $15.5905 \pm 0.0008$ \\
Gaia $G_{\text {BP }}$ & $18.4877 \pm 0.0182$ & $17.0644 \pm 0.0071$ & $15.9241 \pm 0.0061$ \\
Gaia $G_{\text {RP }}$ & $18.2951 \pm 0.0215$ & $16.7969 \pm 0.0125$ & $15.1300 \pm 0.0024$ \\
2MASS $J$ & - & $16.836 \pm 0.142$ & $14.939 \pm 0.037$ \\
2MASS $H$ & - & - & $14.728 \pm 0.045$ \\
2MASS $K$ & - & - & $14.706 \pm 0.080$ \\
UKIDSS $Y$ & - & $16.917 \pm 0.010$ & - \\
UKIDSS $J$ & - & $16.848 \pm 0.014$ & - \\
UKIDSS $H$ & - & $16.864 \pm 0.045$ & - \\
UKIDSS $K$ & - & $16.661 \pm 0.052$ & - \\
WISE $W 1$ & $18.324 \pm 0.124$ & $16.788 \pm 0.128$ & $14.569 \pm 0.015$ \\
WISE $W 2$ & - & & \\
\hline & & &
\end{tabular}

Table 2. Observing log for $H S T$ spectra for the three DZs. $N_{\text {orbit }}$ is the number of orbits per object, and $t_{\mathrm{exp}}$ is the combined integration time for all orbits.

\begin{tabular}{lccc}
\hline SDSS J & Obs. Date & $N_{\text {orbit }}$ & $t_{\exp }$ \\
\hline $0956+5912$ & $2016-04-20$ & 2 & 5684 \\
$1038-0036$ & $2016-05-15$ & 2 & 5225 \\
$1535+1247$ & $2016-08-11$ & 4 & 10608 \\
\hline
\end{tabular}

in Table 2. Table 1 shows that synthetic GALEX NUV photometry calculated from the STIS data are consistent with the values measured by GALEX itself.

\section{DZ MODELS AND SPECTRAL ANALYSIS}

The three white dwarfs were refitted using Koester (2010) model atmospheres, though with improvements to the code compared with our previous analyses of these stars. In Hollands et al. (2017), we presented new calculations for unified line profiles for the $\mathrm{Ca}$ II $\mathrm{H}+\mathrm{K}$ doublet, $\mathrm{Mg}$ II $\mathrm{h}+\mathrm{k}$ doublet, and $\mathrm{Mg}_{\mathrm{I}} \mathrm{b}$ triplet, though the latter was not used in our previous analysis due to concerns over the atmospheric conditions for this triplet potentially exceeding the validity of the unified theory, instead using the more approximate quasi-static profiles (Walkup et al. 1984).

Since then similar calculations for the Mg triplet have been used (Blouin 2020) to show that the quasi-static profiles underestimate the atmospheric abundances, potentially solving an $\mathrm{Mg}$ under-abundance issue identified by in the Hollands et al. (2017) sample by Turner \& Wyatt (2020). We therefore also use our most up-to-date version of the $\mathrm{Mg}$ triplet unified-profiles in this work. Furthermore, our models now include a Ca-He unified profile for the $\mathrm{Ca}$ I $4227 \AA$ resonance line, first used by Hollands et al. (2021).

We also included photo-ionisation cross-sections (from TOPbase; Cunto et al. 1993) for dominating metals into both the atmospheric structure calculations and spectral synthesis. We initially experimented to see which cross sections were most important, so that those making effectively no detectable difference were excluded during the fittings procedure. For all three DZs, we used cross-sections for Na I, $\mathrm{Mg}_{\mathrm{I}}, \mathrm{Al}_{\mathrm{I}}, \mathrm{Si}$ I, Ca I-II, and Fe I, though for SDSS J0956+5912 C $\mathrm{I}$ was also necessary.

Compared to the optical, the NUV contains a higher density of metal absorption lines, many of which are broad and near saturated. Therefore an an increased number of wavelength points were used in the atmospheric structure calculation, in order to correctly sample the opacity contribution from all strong lines.

The models were fitted directly to the flux-calibrated spectra using a $\chi^{2}$-minimization routine, with $T_{\text {eff }}$, white dwarf radius, and abundances of all detected elements as free parameters at each step recalculating the model structure and synthetic spectrum for selfconsistent results. Previously measured atmospheric parameters were initialised using the solutions from Hollands et al. (2017). For newly identified elements (where lines were all weak lines), initial abundances were adjusted manually until reasonable visual agreement was found. For each object all available spectra were used in the fit, with the models convolved to the instrumental resolution appropriate to each spectrum, and with their individual $\chi^{2}$ summed to 
provide the final $\chi^{2}$ for minimization. The radii were constrained via the Gaia parallaxes, and converted to surface gravities for input to the model atmospheres using a mass-radius relation appropriate for thin hydrogen-layers (Bédard et al. 2020). For SDSS J1038-0036 and SDSS J1535+1247 where hydrogen lines are not observed, we adopted the approach of Coutu et al. (2019), where hydrogen abundances were fixed to the detection-limit. This approach has been found to result in DZ masses more consistent with the mass distribution found for other white dwarf spectral types (Coutu et al. 2019). Since these stars are within $150 \mathrm{pc}$ of the Sun, interstellar reddening $E(B-V)<0.01$ in all cases (Green et al. 2018, 2019), and so was assumed to be negligible.

The scaling of the models to the spectroscopic data in the continuum was used to determine white dwarf angular sizes and their uncertainties since these are anti-correlated with $T_{\text {eff }}$. Combining with the parallaxes and their errors allowed us to measure the white dwarf radii and its covariance with $T_{\text {eff }}$. Finally, we used a white dwarf mass-radius relation (Bédard et al. 2020) to determine the surface gravities and masses as well as their uncertainties.

To estimate parameter uncertainties, we calculated onedimensional model grids around the derived solution, in steps of $50 \mathrm{~K}$ for $T_{\text {eff }}$, and steps of 0.1 dex for abundances. These models were then visually compared against all spectroscopic data to estimate the level of uncertainty for each parameter. A caveat of this approach, is that it does not consider covariance between parameters, and so will lead to underestimates for some abundances, notably between $T_{\text {eff }}, \mathrm{H}, \mathrm{Mg}, \mathrm{Si}, \mathrm{Ca}$, and $\mathrm{Fe}$, which are typically mutually correlated. Inspection of the parameter correlation matrices (derived from the covariance matrices of our fits) indicates that this is at most a factor $\mathrm{few}^{3}$. Specifically $\mathrm{Ca}$ abundance uncertainties should be scaled by about a factor four, whereas a factor two is more appropriate for $\mathrm{H}$, $\mathrm{Mg}, \mathrm{Al}, \mathrm{Si}, \mathrm{Mn}, \mathrm{Fe}$, and $\mathrm{Ni}$; or in other words, for most elements uncertainties including covariances are at least 0.2 dex. The remaining elements we have fitted $(\mathrm{C}, \mathrm{O}, \mathrm{Na}, \mathrm{Ti}$, and $\mathrm{Cr}$ ) only change the emergent flux where a limited set of narrow lines are present, and thus are not correlated with other parameters.

The results to our fits are listed in Table 3, with the best fitting models shown against all spectra in Figure 1. In all three panels, the models adequately reproduce the spectral energy distributions, both in the optical and the NUV. Nevertheless, a few discrepancies remain. In particular, some missing absorption is observed in the spectra of SDSS J0956+5912 and SDSS J1038-0036 in a narrow wavelength range around $\approx 2013 \AA$. Searching with the NIST atomic spectra line database form (Kramida et al. 2016), we did not find any convincing candidates for missing absorption lines. The Co II resonance line at $2012.2 \AA$ seemed the most likely choice, but would require other strong Co II lines to be visible if this ion was present in the atmospheres of SDSS J0956+5912 and SDSS J1038-0036. Other minor discrepancies are visible at NUV wavelengths between regions of strong line absorption (particularly for SDSS J1038-0036). However, because these differences only affect a tiny fraction of the emergent flux, their influence on our derived atmospheric parameters can be considered to be negligible.

\section{EFFECTIVE TEMPERATURES}

Our primary objective in this study was to assess the accuracy of the atmospheric parameters derived from Hollands et al. (2017),

\footnotetext{
3 Scaling factors to correct the 1D error estimates can be found by taking the square roots of the diagonal elements of the inverse correlation matrices.
}

when including the NUV range in addition to only using the optical. For SDSS J1038-0036 and SDSS J1535+1247, our $T_{\text {eff }}$ and abundance measurements were found to be consistent with the results of Hollands et al. (2017), whereas for SDSS J0956+5912 we found a substantially lower temperature once the NUV was included (Table 3). Indeed our earlier model from Hollands et al. (2017) shows the NUV flux level to be about 2-3 times higher than the flux observed with STIS. This poses the question as to what is special about SDSS J0956+5912 in comparison to SDSS J1038-0036 and SDSS J1535+1247 that such a large difference was found. We suggest some potential sources for this systematic uncertainty affecting SDSS J0956+5912, though we do not find a conclusive explanation.

All three objects were observed with the original SDSS spectrograph, with a wavelength range extending as blue as $3800 \AA$. However, in the Hollands et al. (2017) analysis of SDSS J1038-0036 and SDSS J1535+1247 we had obtained additional WHT spectra covering wavelengths down to $3100 \AA$. In all three spectra (Figure 1), a significant amount of flux is observed between $3000-3900 \AA$ essentially the range between the extremely strong $\mathrm{Mg}_{\text {II }} \mathrm{h}+\mathrm{k}$ and $\mathrm{Ca}$ II $\mathrm{H}+\mathrm{K}$ doublets. Therefore in the analysis of Hollands et al. (2017) this wavelength interval was already well constrained for SDSS J1038-0036 and SDSS J1535+1247, but practically unconstrained for SDSS J0956+5912 (this wavelength range is now largely covered by our GTC $u$-band spectrum). Even so, we only found small differences between the flux levels in the $u$-band between the older and newer models (where the main difference is instead the NUV as described above). Though, we note that compared to our model from Hollands et al. (2017), the wings of the $\mathrm{Ca} \mathrm{H}+\mathrm{K}$ resonance lines are in better agreement with the optical spectra, and the Mg II $4481 \AA$ line is also better matched in strength.

One difference which primarily affects SDSS J0956+5912 between our model from Hollands et al. (2017) and our new results, is the inclusion of new elements that were not previously detected. In particular $\mathrm{Al}$ is identified from a $\mathrm{Al}$ I resonance doublet that sits between the $\mathrm{Ca} \mathrm{H}+\mathrm{K}$ lines. While the doublet itself makes only a minor difference to the emergent flux of the spectrum, the continuous opacity contributed by this element's photoionization cross-section is considerable between between 1950-2250 A, reducing the emergent flux by half (compared to when the continuous opacity is excluded). However, this can not explain the difference in $T_{\text {eff }}$ we have found as excluding this opacity source would necessitate an even lower $T_{\text {eff }}$ in order to remain broadly consistent with the observed fluxes.

Other recent analyses using similarly modern white dwarf model atmosphere codes find virtually the same solution as the Hollands et al. (2017) results when using only the SDSS spectrum. Making use of the then newly available Gaia DR2 parallaxes, Coutu et al. (2019), fitted the spectrum of SDSS J0956+5912, assuming bulk Earth abundance ratios between metals, finding $T_{\text {eff }}=8723 \pm 105 \mathrm{~K}$, and a $\mathrm{Ca}$ abundance of $\log (\mathrm{Ca} / \mathrm{He})=-7.12 \pm 0.09$. More recently Blouin (2020) revisited the Hollands et al. (2017) DZ sample using their model atmosphere code. They elected to pre-compute a 6-dimensional grid of models $\left(T_{\text {eff }}, \log g ; \mathrm{H}, \mathrm{Ca}, \mathrm{Mg}\right.$, and Fe abundances) to use in their analysis, and found $T_{\text {eff }}=8843 \pm 180 \mathrm{~K}$, again highly similar to our previous results. While these analyses find similar results to our previous solution using optical spectra, we naturally cannot assess how these different models would perform against the NUV data presented here. It is also important to note that the models used by Coutu et al. (2019) and Blouin (2020), will contain some differences in constituent physics such as the equation of state, ionisation equilibrium, and unified line profiles used for the strongest metal lines (Blouin et al. 2018), any of which could plausibly lead to differences in results when analysing stars with extreme abundances, 
Table 3. Atmospheric parameters derived in this work (H2021), compared with previous results from Hollands et al. (2017) (H2017). Abundances are logarithmic number abundances relative to helium, e.g. Ca corresponds to $\log (\mathrm{Ca} / \mathrm{He})$, and where their reported uncertainties do not include correlations between parameters.

\begin{tabular}{lcccccc}
\hline SDSS J & $0956+5912$ & \multicolumn{2}{c}{$1038-0036$} & \multicolumn{2}{c}{$1535+1247$} \\
\hline & $\mathrm{H} 2021$ & $\mathrm{H} 2017$ & $\mathrm{H} 2021$ & $\mathrm{H} 2017$ & $\mathrm{H} 2021$ & $\mathrm{H} 2017$ \\
\hline$T_{\text {eff }}[\mathrm{K}]$ & $8100 \pm 100$ & 8800 & $7560 \pm 150$ & 7700 & $5680 \pm 50$ & 5773 \\
$\log g$ & $8.02 \pm 0.04$ & 8.00 & $8.06 \pm 0.04$ & 8.00 & $8.06 \pm 0.03$ & 8.00 \\
$M\left[\mathrm{M}_{\odot}\right]$ & $0.59 \pm 0.02$ & - & $0.61 \pm 0.03$ & - & $0.61 \pm 0.02$ & - \\
$\mathrm{H}$ & $-3.2 \pm 0.1$ & -3.6 & $\leq-5.0$ & $\leq-4.8$ & $\leq-2.5$ & -3 \\
$\mathrm{C}^{a}$ & {$[-5.2,-4.1]$} & - & - & - & - & - \\
$\mathrm{O}^{b}$ & $-4.1 \pm 0.2 /-4.6 \pm 0.1$ & - & - & - & - & - \\
$\mathrm{Na}$ & $-6.8 \pm 0.2$ & -6.60 & $-8.7 \pm 0.1$ & -8.60 & $-8.85 \pm 0.05$ & -8.72 \\
$\mathrm{Mg}$ & $-5.5 \pm 0.1$ & -5.30 & $-6.6 \pm 0.1$ & -6.80 & $-6.9 \pm 0.1$ & -7.36 \\
$\mathrm{Al}$ & $-6.5 \pm 0.1$ & - & - & - & $-8.4 \pm 0.4$ & - \\
$\mathrm{Si}$ & $-5.7 \pm 0.2$ & - & $-6.4 \pm 0.2$ & - & $-6.5 \pm 0.3$ & - \\
$\mathrm{Ca}$ & $-7.30 \pm 0.05$ & -7.15 & $-7.60 \pm 0.05$ & -7.85 & $-8.50 \pm 0.05$ & -8.61 \\
$\mathrm{Ti}$ & $-8.9 \pm 0.2$ & - & $-9.5 \pm 0.1$ & -9.60 & $-10.2 \pm 0.3$ & -9.62 \\
$\mathrm{Cr}$ & - & -7.50 & - & - & $-9.6 \pm 0.3$ & -9.25 \\
$\mathrm{Mn}$ & $-9.2 \pm 0.2$ & - & $-9.2 \pm 0.2$ & - & $-10.0 \pm 0.1$ & - \\
$\mathrm{Fe}$ & $-6.9 \pm 0.1$ & -6.14 & $-7.4 \pm 0.1$ & -7.40 & $-7.6 \pm 0.1$ & -7.57 \\
$\mathrm{Ni}$ & - & - & $-8.6 \pm 0.1$ & - & $-8.6 \pm 0.2$ & -8.90 \\
\hline
\end{tabular}

Notes: (a) The carbon abundance is given as a lower/upper-limit pair based on the flux-suppression in the NUV and absence of molecular features in the optical. (b) The oxygen abundance pair corresponds to the SDSS/GTC spectra respectively. (c) A noise spike in the SDSS spectrum of SDSS J0956+5912 was previously misidentified as the $5207 \AA$ Ar I triplet, however the absence of other strong $\mathrm{Cr}$ I-II lines rules out the detection of this element.

as is the case for SDSS J0956+5912. However, direct comparisons between these model atmosphere codes is beyond the scope of this work.

\section{CARBON DETECTION IN SDSS J0956+5912}

For SDSS J0956+5912 we report a tentative detection of photospheric carbon based on the $1931 \AA$ line in the HST spectrum. In Fig. 2, we show the NUV spectrum of SDSS J0956+5912 alongside two models. The solid red curve corresponds to a model with $\log (\mathrm{C} / \mathrm{He})=-4.5 \mathrm{dex}$, whereas the dotted curve corresponds to a model without any carbon. Evidently the carbon-rich model provides a better fit in the range 1910-1940 ̊ where more flux would be expected in the absence of carbon. Transitions from other elements were not found to be plausible, as the only other strong lines (excluding trans-iron elements) in this range belong to elements already measured at other wavelengths $(\mathrm{Ti}, \mathrm{Mn}, \mathrm{Fe})$, but at far too small abundances to cause strong absorption in the 1910-1940 ̊ range. Indeed the intrinsically strong lines from these other elements were already included in our line list for both models in Figure 2.

Detection of both carbon and heavy metals in white dwarf envelopes is rare, though not unprecedented. Examples include Procyon B which was classified as a DQ from its optical spectrum, though was reappraised as a DQZ following the identification of magnesium from the $\mathrm{Mg}$ II resonance lines in its UV spectrum (Provencal et al. 2002). Three further DQZ stars have been identified more recently by Coutu et al. (2019). For objects classed DZQ, i.e. where metal lines are dominant and carbon features are secondary, we have only been able to identify one object in the literature: L 119-34 (WD 2216-657) as identified by Wolff et al. (2002). Similarly to SDSS J0956+5912 L 119-34 has an optical spectrum dominated by metal lines (Swan et al. 2019), but with the $1931 \AA \mathrm{C}_{\mathrm{I}}$ line also present in the UV as demonstrated by its IUE spectrum.

However we acknowledge there are some reasons to treat this detection as tentative. Firstly, this is the only carbon feature observed, without additional corroborating evidence across the spectrum. Secondly, several regions in the NUV are poorly modelled, and so it is feasible that the $1910-1940 \AA$ range simply represents one more such region.

Assuming carbon is indeed present in the atmosphere, this element could have either been accreted alongside the metals accreted by SDSS J0956+5912, or could be present due to dredge up of carbon from the core, i.e. SDSS J0956+5912 is a former DQ white dwarf since converted to a DZQ. The first scenario can be considered unlikely as the implied quantity of carbon is so large, that it dwarfs all other metals except for oxygen (20-40 percent of the total metal mass). This allows for a roughly one-to-one carbon-to-oxygen ratioinconsistent with other metal-rich white dwarfs where carbon abundances have been explored (Wilson et al. 2016). In the dredge up scenario, the carbon abundance we found is certainly consistent with other DQs with the same $T_{\text {eff }}$ (Koester \& Kepler 2019; Coutu et al. 2019; Blouin \& Dufour 2019). However the DQs within the published literature are predominantly identified via molecular features from $\mathrm{C}_{2}$ in the optical, yet these features are not seen for SDSS J0956+5912. Thus we considered the possibility that the presence of metals could reduce the observed strength of the Swan bands, preventing the detection of carbon in DZs. We therefore constructed a small grid of models as a function of metal abundance, starting with our best fit model to the SED of SDSS J0956+5912 and successively reducing metal abundances in 1 dex steps, down to $\log \left(Z / Z_{0956}\right)=-4$ (where $Z$ is the abundance of any metal and with the carbon abundance held constant at $\log (\mathrm{C} / \mathrm{He})=-4.5$, and $Z_{0956}$ is the corresponding abundance found for SDSS J0956+5912). The full grid of models are shown in Figure 3.

As hypothesised above, as the metal abundances are reduced, the Swan bands become visible reaching their full strength at $\log \left(Z / Z_{0956}\right) \approx-3$. In addition, other carbon features become visible in different parts of the spectrum. The asymmetric $2479 \AA^{\circ} \mathrm{C}_{\mathrm{I}}$ line rapidly becomes apparent as metals are reduced allowing it to be distinguished from the forest of metal lines. At around $3145 \AA$ 


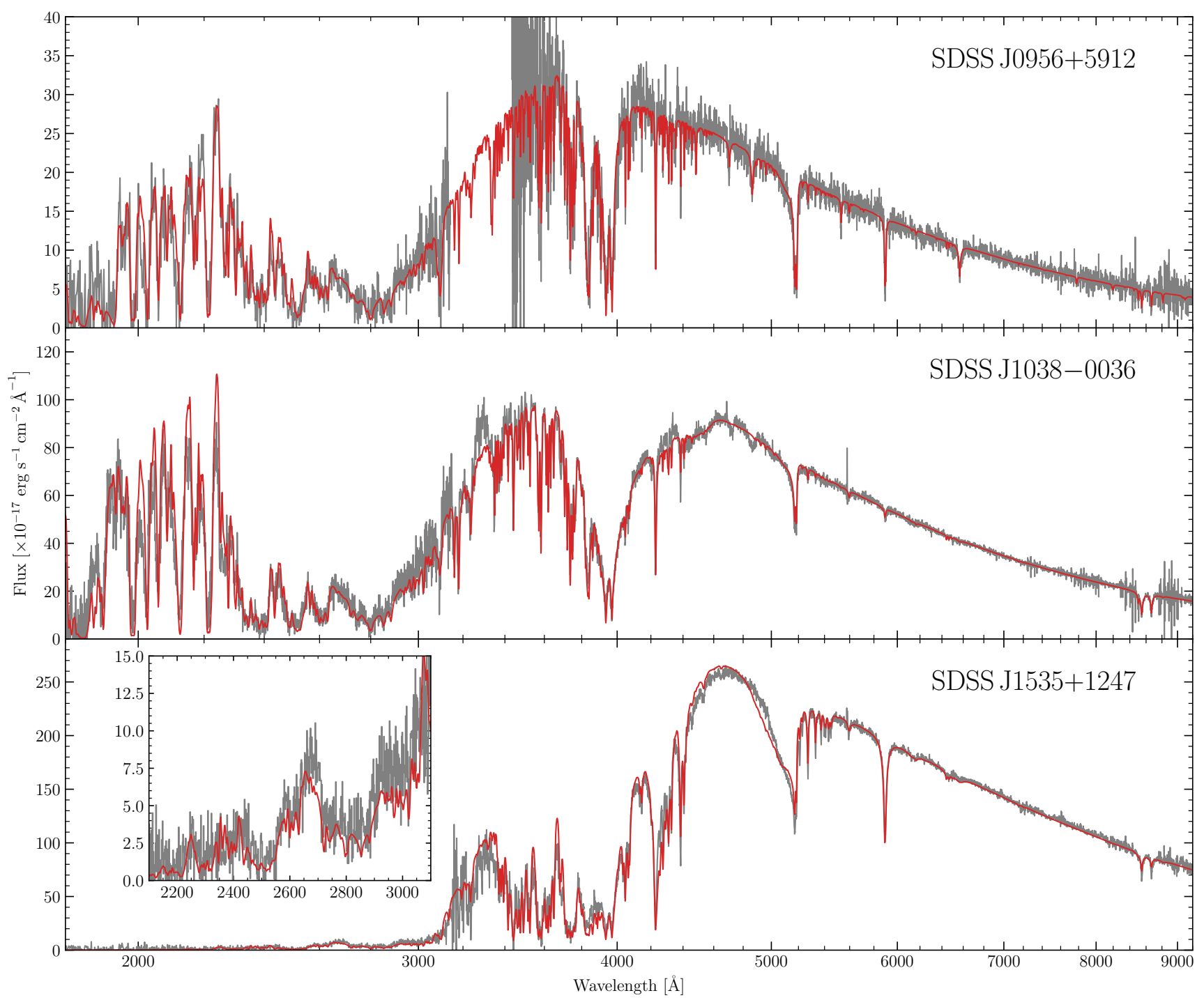

Figure 1. Spectral energy distributions of all three white dwarfs covering both the NUV and optical with their best fitting models (red). For SDSS J1535+1247 the insert shows the diminished flux in the NUV.

the $\mathrm{CH}$ molecular band also becomes visible, appearing weakly for $\log \left(Z / Z_{0956}\right) \approx-1$, and then much more strongly as metal abundances are reduced further. An additional $\mathrm{CH}$ feature is seen at $4310 \AA$, but it is much weaker, and is instead comparable in strength to the Swan bands.

We considered the possibility that the presence of metals provides more free electrons to the atmosphere lowering the molecular number density at equilibrium. However inspecting our models as a function of optical depth, we found that the molecular abundances were not significantly changed. Instead we found that at the high metal abundances of SDSS J0956+5912 the opacity from metals simply dominated the molecular opacity of carbon and so neither $\mathrm{CH}$ nor $\mathrm{C}_{2}$ molecular bands could be detected.

DZ and DQ white dwarfs both correspond to at least $15 \pm 4$ percent of the local population with helium-rich atmospheres (McCleery et al. 2020) ${ }^{4}$. If the presence of carbon and metals are independent, about 15 percent of all DZ white dwarfs (corresponding to 2.25 percent of white dwarfs with helium-rich atmospheres) would be expected to show carbon lines, e.g. DZQ/DQZ spectral types. Large observed samples of DZ stars contain a much lower fraction of DZQs/DQZs (Coutu et al. 2019), illustrating the dearth of these objects.

It is tempting to attribute the changing carbon feature strengths as a function of metal abundance as an explanation for the apparent absence of known DZQs, i.e. that the accretion of sufficiently large amounts of planetary material can convert a DQ directly to a DZ (when only optical spectra are considered). While this is a possibility for SDSS J0956+5912 and other DZs with extreme metal-pollution, this cannot explain the absence of DZQs/DQZs in a more general

\footnotetext{
${ }^{4}$ Ratio based on confirmed helium-rich atmospheres with $T_{\text {eff }}>5000 \mathrm{~K}$ and medium resolution spectroscopy in the northern hemisphere $40 \mathrm{pc}$ sample.
} 


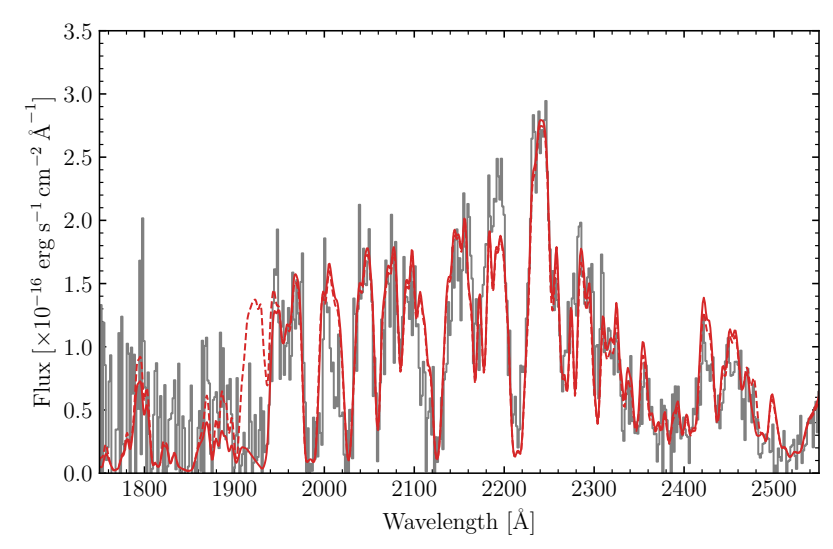

Figure 2. NUV spectrum of SDSS J0956+5912 (grey). The best fitting model and a similar model with carbon removed are shown by the red solid/dashed curves, respectively.

scenario. Figure 3 shows that at $\log \left(Z / Z_{0956}\right)=-3$, when the $C_{2}$ bands reach their peak strength, the $\mathrm{Ca} \mathrm{H}+\mathrm{K}$ lines in the optical and $\mathrm{Mg} \mathrm{h}+\mathrm{k}$ lines in the NUV still remain visible at a detectable level. Therefore a DQ accreting small traces of rocky material would undoubtedly be identifiable as a DQZ/DZQ, as was the case for Procyon B (as mentioned above, Provencal et al. 2002).

\section{THE PLANETESIMAL ACCRETED BY SDSS J0956+5912}

\subsection{Silicon and oxygen detections}

SDSS J0956+5912 was previously reported as an outlier in terms of its large magnesium abundance by Hollands et al. (2018). Assuming this magnesium to be part of silicate rich material suggested silicon and oxygen should also be present in similarly large quantities. For all three systems, the HST data provided coverage of detectable Si I absorption. The strongest groups of (blended) lines are found near $1900 \AA, 2060 \AA, 1980 \AA, 2129 \AA, 2210 \AA$, and $2520 \AA$. However, for SDSS J0956+5912, we found that a further constraint on the $\mathrm{Si}$ abundance was provided by the optical $3905 \AA$ line which we had previously missed. ${ }^{5} \mathrm{Re}$-inspection of all spectra included in Hollands et al. (2017) did not reveal the Si I $3905 \AA$ line at any of the other 230 white dwarfs in that work regardless of the spectral signal-to-noise ratio, leaving only SDSS J0956+5912 with this line present.

The measured silicon abundance is comparable to magnesium (Table 3). Within rocky material, these elements are typically part of the metal oxides $\mathrm{MgO}$ and $\mathrm{SiO}_{2}{ }^{6}$, thus suggesting around three times as much oxygen present compared with magnesium. Unlike Si I, there are no strong $\mathrm{O}_{\mathrm{I}}$ lines in the NUV, only in the optical, where a blend of three lines centred on $7775 \AA$ (including the $\sim 80 \mathrm{~km} \mathrm{~s}^{-1}$ redshift measured from other photospheric lines), ought to be the strongest $\mathrm{O}$ I feature at this $T_{\text {eff }}$. Indeed, a weak, but clear $\mathrm{O}_{\mathrm{I}}$ feature is detected at $7775 \AA$ in the SDSS spectrum of SDSS J0956+5912, corresponding to a measured abundance of $\log (\mathrm{O} / \mathrm{He})=-4.1 \pm 0.2 \mathrm{dex}-$ much

\footnotetext{
5 While Si was not explicitly fitted in Hollands et al. (2017), lines from this element were still included in the modelling but with abundances fixed relative to $\mathrm{Ca}$. In hindsight, scaling $\mathrm{Si}$ with $\mathrm{Mg}$ would have been a better choice.
}

higher than can be expected for a combination of the oxides $\mathrm{MgO}$ and $\mathrm{SiO}_{2}$.

To better measure the oxygen abundance, we obtained a higher quality spectrum with the OSIRIS instrument on the GTC, and using the $i$-band VPH grating (Section 2.1). The $\mathrm{O}_{\text {I }}$ blend remains visible in the GTC spectrum, though surprisingly with the line strength reduced compared to the SDSS spectrum (Figure 4), and the corresponding abundance diminished to $\log (\mathrm{O} / \mathrm{He})=-4.6 \pm 0.1 \mathrm{dex}$ (Table 3). We do not observe similar changes in line strength for other transitions covered by both the SDSS and GTC spectra. In the GTC $i$-band spectrum no change is seen for the Ca II triplet, nor the $8807 \AA \mathrm{Mg}$ I line, compared with the SDSS spectrum. Furthermore, the approximately twenty narrow absorption lines observed in both the GTC $u$-band spectrum and SDSS spectrum are also consistent in strength.

To precisely quantify the apparent change in oxygen line strength, we estimated the equivalent widths in both spectra, adopting an approach based on the optimal-extraction algorithm developed for CCD spectroscopy (Horne 1986). This method essentially performs a weighted sum across a row of pixels, where the weights are chosen to maximise the sum signal to noise ratio. This has the advantage of ignoring the contribution of variance far outside the line-profile, as would be the case with a straight sum. One important ingredient to this approach is that an accurate shape of the line-profile must be known in advance, which fortunately is possible in our case. We extract this shape directly from the model spectrum, convolving to the instrumental resolution, and redshifting to the frame as measured from other spectral lines.

Indeed the equivalent widths were found to be in disagreement (Figure 4), where the line strengths are seen to change by $1.66 \pm 0.57 \AA$, i.e. about $3 \sigma$ difference. We are unable to offer a clear explanation for why the oxygen triplet appears to have changed strength. As previously mentioned, the moderate $80 \mathrm{~km} \mathrm{~s}^{-1}$ velocityshift of the oxygen triplet matches that of the other metal lines in the spectrum confirming it is of photospheric origin. While a weak magnetic field combined with rotation could plausibly lead to line strength variability, we do not observe similar variation between other lines. Furthermore, no evidence of Zeeman splitting is observed in any lines, in particular the components of the $\mathrm{Ca}$ II infra-red triplet which is particularly sensitive to small fields due to its red wavelength and large Landé- $g$ factors, allowing us to place an upper-limit of $40 \mathrm{kG}$ from the GTC $i$-band spectrum. A heterogeneous abundance distribution is also ruled out as the horizontal spreading timescale for metals across the surface of cool white dwarfs with helium-dominated atmospheres should be around four orders of magnitude faster than the sinking timescale (Cunningham et al. 2021). With no ready explanation for the peculiar change in abundances, we conduct the rest of our analysis into SDSS J0956+5912 referring to results for both the SDSS and GTC abundances.

While substantially lower than for the SDSS spectrum (-4.1 dex), the GTC abundance $(-4.6 \mathrm{dex})$ remains larger than expected for a composition dominated by $\mathrm{MgO}$ and $\mathrm{SiO}_{2}$. Given that the other metals in SDSS J0956+5912 are essentially traces compared with magnesium, silicon, and oxygen (for instance iron is about 1 dex depleted relative to its bulk Earth ratio compared with magnesium), it

\footnotetext{
6 The base metal oxides $\mathrm{MgO}$ and $\mathrm{SiO}_{2}$ needn't be present in this form prior to accretion, and could instead by part of more complex minerals such as $\mathrm{MgSiO}_{3}$ (enstatite) or $\mathrm{Mg}_{2} \mathrm{SiO}_{4}$ (forsterite). Since we only measure the individual atomic abundances, we refrain from further speculation on the precise mineralogy of the accreted parent body and instead refer to the base metal oxides.
} 


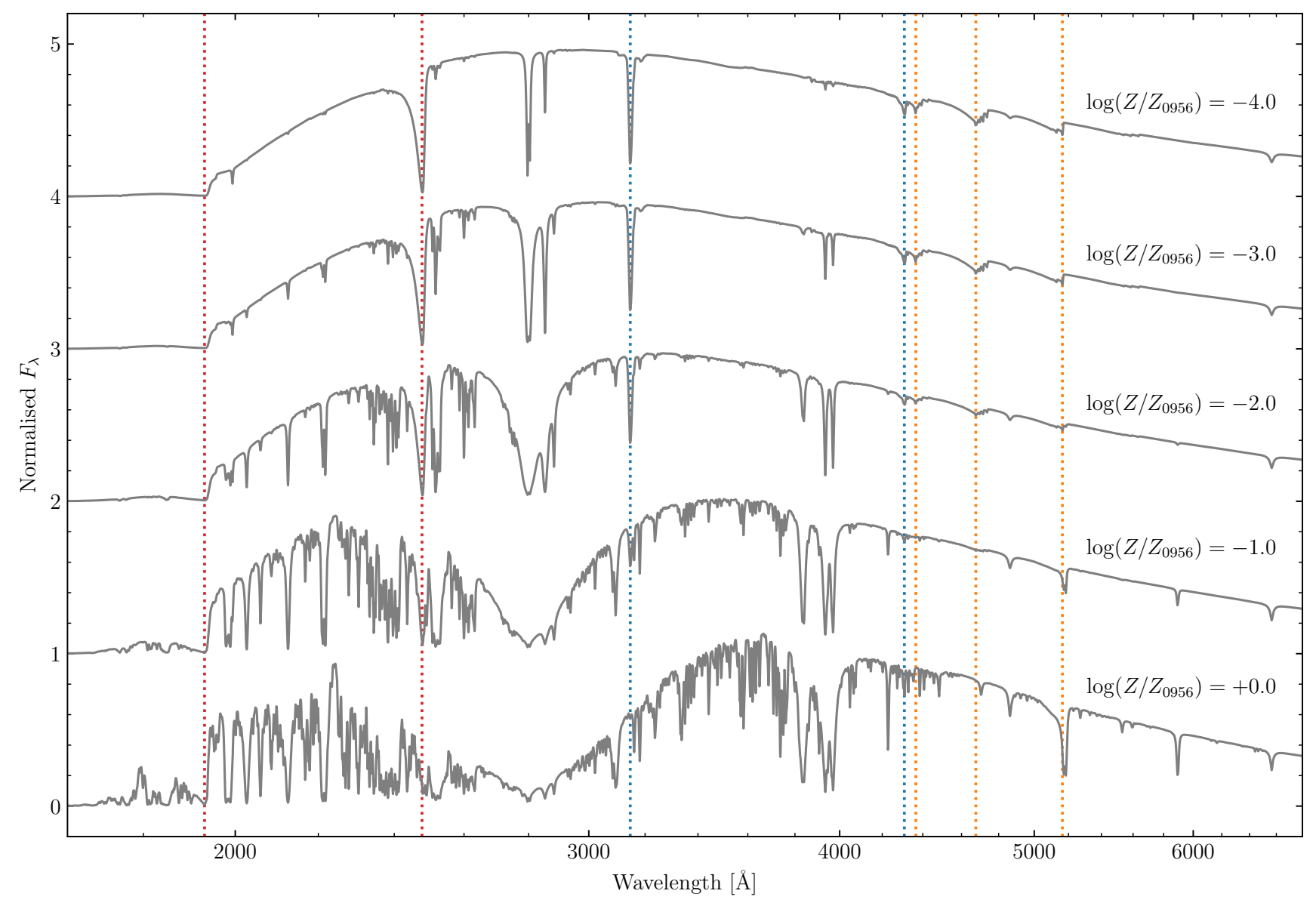

Figure 3. Grid of models starting at the measured abundances of SDSS J0956+5912, removing each metal (excluding carbon) in 1 dex steps (bottom to top). As metals are removed, molecular carbon features become increasingly visible. The carbon abundance is set to $\log (\mathrm{C} / \mathrm{He})=-4.5 \mathrm{in}$ all cases. Wavelengths for atomic carbon, $\mathrm{CH}$ molecules, and $\mathrm{C}_{2}$ molecules are indicated by the red, blue, and orange dotted lines, respectively.

is clear that this oxygen over-abundance cannot be entirely attributed to other metal oxides. Past work has shown that oxygen-excesses can be explained as water making up a large fraction of the accreted material (Farihi et al. 2013; Raddi et al. 2015). In those reported cases, the white dwarfs are of spectral type DBZA, where the trace hydrogen serves as an additional signature of water accretion (Gentile Fusillo et al. 2017), though unlike metals hydrogen does not sink out of the white dwarf envelope, instead accumulating over all accretion episodes. Like those previous cases, SDSS J0956+5912 also shows hydrogen traces, at an abundance of $\log (\mathrm{H} / \mathrm{He})=-3.2 \pm 0.1 \mathrm{dex}$. The water accretion interpretation will be discussed in more detail in Section 6.4.

\subsection{Envelope parameters of SDSS J0956+5912}

To fully analyse the composition of the accreted material requires knowledge of the white dwarf envelope parameters, specifically the convection zone mass and the diffusion timescales for each element in the atmosphere. The extreme metal abundances (as well as the large quantity of trace hydrogen) have a strong affect on the atmospheric structure, and in turn upon the envelope parameters. It is therefore insufficient to use a tabulated grid of values as function of $T_{\text {eff }}$ and $\log g$, where trace metal abundances have little effect on the structure, and instead bespoke calculations are required. We calculated these convection zone parameters using the latest version of the Koester envelope code (Koester et al. 2020), using our best fitting model for SDSS J0956+5912 as a boundary condition at the top of the envelope. We calculated two envelope models, firstly with the chemically mixed region determined purely from mixing length theory, and secondly with convective-overshoot extending the chemically mixed region by one pressure scale height (results presented in Table 4). The effect of this overshoot acts to increase diffusion timescales by 0.35 dex or a factor of 2.2. Compared to models with only minute traces of metals (but the same $T_{\text {eff }}$ and $\log g$ ), the bespoke models result in smaller convection zones (by $0.67 \mathrm{dex}$ ), and consequently also have shorter diffusion timescales (by about $0.6 \mathrm{dex}$ ).

\subsection{An oxygen excess}

We apply the same analysis method as that used by Farihi et al. (2013) to quantify the level of excess oxygen. Specifically, we assume metals heavier than oxygen ${ }^{7}$ were originally in metal oxide form, e.g. $\mathrm{MgO}, \mathrm{Al}_{2} \mathrm{O}_{3}$, and $\mathrm{SiO}_{2}$. The corresponding amounts of oxygen are then subtracted from the total oxygen budget revealing any remaining oxygen as an excess. However, depending at which stage of accretion SDSS J0956+5912 is observed at, different treat- 

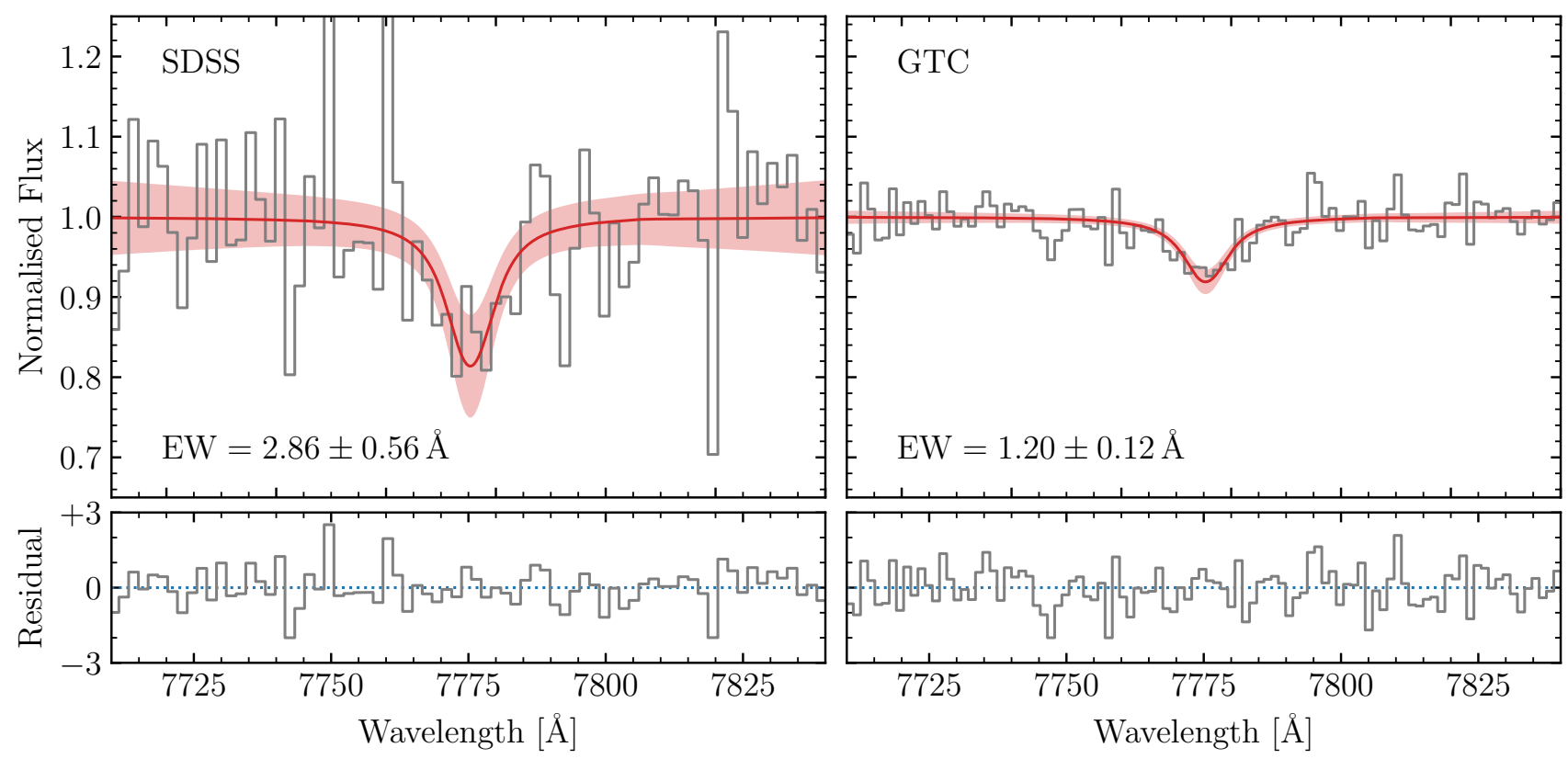

Figure 4. Equivalent width of the O I blend for SDSS J0956+5912, as measured from the SDSS and GTC spectra. The red shaded regions indicate the $2 \sigma$ confidence region around the best fit. The residuals between the data and fit are scaled by the data uncertainties.

Table 4. Envelope parameters for SDSS J0956+5912. The first and second sets of columns correspond to envelope models calculated with and without convective overshoot extending the size of the convection zone by one pressure scale height, respectively. $\log q$ is the logarithm (base-10) of the fractional convection zone mass, $M_{\mathrm{cvZ}} / M_{\mathrm{wd}}$. Diffusion timescales for each element, $\tau_{Z}$ are in units of years; the convection zone masses for each element, $m_{Z}$, in $\mathrm{g}$; and the diffusion fluxes out of the base of the convection zone, $\dot{m}_{Z}$, in $\mathrm{g} \mathrm{s}^{-1}$ (with all three quantities expressed as base-10 logarithms). Oxygen masses and diffusion fluxes correspond to the abundance as measured in the SDSS spectrum, where the corresponding values for the GTC spectrum can be obtained by subtracting 0.50 dex. Diffusion timescales were calculated for nickel, although since this element is not detected, values of $m_{Z}$, and $\dot{m}_{Z}$ cannot be calculated.

\begin{tabular}{|c|c|c|c|c|c|c|}
\hline \multirow[t]{2}{*}{$\log q$} & \multicolumn{3}{|c|}{$\begin{array}{l}\text { Overshoot } \\
-5.224\end{array}$} & \multicolumn{3}{|c|}{$\begin{array}{c}\text { No overshoot } \\
-5.658\end{array}$} \\
\hline & $\log \tau_{Z}$ & $\log m_{Z}$ & $\log \dot{m}_{Z}$ & $\log \tau_{Z}$ & $\log m_{Z}$ & $\log \dot{m}_{Z}$ \\
\hline $\mathrm{H}$ & - & 24.05 & - & - & 23.61 & - \\
\hline $\mathrm{C}$ & 6.460 & 23.82 & 9.87 & 6.114 & 23.39 & 9.78 \\
\hline $\mathrm{O}$ & 6.375 & 24.35 & 10.48 & 6.026 & 23.91 & 10.39 \\
\hline $\mathrm{Na}$ & 6.239 & 21.80 & 8.07 & 5.888 & 21.37 & 7.98 \\
\hline $\mathrm{Mg}$ & 6.241 & 23.13 & 9.39 & 5.887 & 22.69 & 9.31 \\
\hline $\mathrm{Al}$ & 6.195 & 22.17 & 8.48 & 5.842 & 21.74 & 8.40 \\
\hline $\mathrm{Si}$ & 6.201 & 22.99 & 9.29 & 5.846 & 22.56 & 9.21 \\
\hline $\mathrm{Ca}$ & 6.088 & 21.55 & 7.96 & 5.730 & 21.11 & 7.88 \\
\hline $\mathrm{Ti}$ & 5.999 & 20.02 & 6.53 & 5.643 & 19.59 & 6.45 \\
\hline $\mathrm{Mn}$ & 5.953 & 19.78 & 6.33 & 5.597 & 19.35 & 6.25 \\
\hline $\mathrm{Fe}$ & 5.956 & 22.09 & 8.64 & 5.599 & 21.66 & 8.56 \\
\hline $\mathrm{Ni}$ & 5.947 & - & - & 5.589 & - & - \\
\hline
\end{tabular}

ments are needed. In the early phase of accretion, diffusion processes have not had time to alter the accreted abundances, and so atmospheric abundance ratios can be taken as reflective of the accreted parent body. If diffusion rates are much shorter than the length of an accretion episode, then a steady-state equilibrium may be reached where accretion balances diffusion processes out of the atmosphere. In this case, the abundances are scaled by dividing by the corresponding diffusion timescales. Finally, we consider the decreasing phase, where a significant fraction of the accreted material may have already sunk out of the outer convection zone. In this case we re- scale each elemental abundance by a factor $\exp \left(t / \tau_{Z}\right)$ where $t$ is the time since accretion (using values of 1,2 , and $5 \mathrm{Myr}$ ), and $\tau_{Z}$ is the diffusion timescale for an element $Z$. Our results from this analysis are shown in Figure 5 for oxygen abundances as measured from both the SDSS and GTC spectra. Note that we do not consider titanium or 
manganese oxides, as Ti and Mn abundances are more than an order of magnitude lower than the other metals that we consider.

It is evident from Figure 5 that in early phase accretion, i.e. using the abundances directly as measured from the atmosphere, SDSS J0956+5912 boasts the largest oxygen excess observed for any white dwarf so far (Farihi et al. 2013; Raddi et al. 2015; Hoskin et al. 2020; Izquierdo et al. 2021), where the excess oxygen fraction is 90 percent for the SDSS measurement, and 70 percent for the GTC spectrum. These excesses are reduced somewhat in the steady-state phase, though such a scenario is unlikely for SDSS J0956+5912 as diffusion timescales in the range 0.9-2.4 Myr are likely to exceed the length of any accretion episode. Furthermore the absence of a flux excess in the WISE photometry suggests the absence of a disc. More significant differences are seen for the decreasing phase, particularly at $5 \mathrm{Myr}$. Here the difference between the $\log (\mathrm{O} / \mathrm{He})=-4.1 /-4.6 \mathrm{dex}$ scenarios becomes vastly more important. For the SDSS measurement, even at $5 \mathrm{Myr}$ the excess remains at $\simeq 70$ percent. By contrast, for the GTC measurement, if accretion occurred $2 \mathrm{Myr}$ ago, a moderate excess of about 45 percent is seen (comparable to the steady-state value for GD 61 as found by Farihi et al. 2013), and as the time since accretion approaches $5 \mathrm{Myr}$, the oxygen-excess is rapidly erased, allowing for a parent body composed almost entirely of metal oxides with little excess oxygen and a total mass of $10^{25} \mathrm{~g}$ (Figure 6). If iron was instead assumed to be present in the form of solid metal then the oxygen fraction $(\simeq 15$ percent at $5 \mathrm{Myr}$ ) previously ascribed to $\mathrm{FeO}$ will correspond to an excess instead (though this excess would also be erased within an additional 1.2 Myr).

\subsection{Evidence for accretion of water ice}

In all previously analysed DBZs where oxygen-excesses have been identified, moderate traces of hydrogen have also been found polluting the white dwarf atmospheres (Farihi et al. 2013; Raddi et al. 2015; Hoskin et al. 2020; Izquierdo et al. 2021). Since the excess oxygen must have been accreted from a solid-component of the parent body, an additional molecule is required, with waterice being the obvious candidate (Gentile Fusillo et al. 2017). For SDSS J0956+5912 a hydrogen abundance of $\log (\mathrm{H} / \mathrm{He})=-3.2$ was measured, and is evidently more than twice the oxygen abundance (i.e. as would be expected for $\mathrm{H}_{2} \mathrm{O}$ ) measured from the SDSS spectrum $(\log (\mathrm{O} / \mathrm{He})=-4.1)$. This difference can be resolved by two possible explanations. If the metals at SDSS J0956+5912 were accreted relatively recently (early phase), then only a small fraction of the observed hydrogen can have been delivered in that accretion episode. This is simply resolved if the remaining hydrogen was delivered from prior accretion episodes in the history of SDSS J0956+5912, where the metals have long since sunk out of the photosphere, but with the integrated hydrogen mass continuing to remain in the white dwarf convection zone. Alternatively, it is possible for all the photospheric hydrogen to have been delivered with the metals if we assume SDSS J0956+5912 is observed far along the decreasing phase. Given the $1.1 \times 10^{24} \mathrm{~g}$ of hydrogen in the white dwarf convection-zone, which does not evolve over time (Figure 6), this necessitates a water mass of $1.0 \times 10^{25} \mathrm{~g}$ within the accreted planetesimal. Specifically, assuming the SDSS abundance,

\footnotetext{
7 We assume any carbon present to be unrelated to the accreted abundances in our analysis, given that $\mathrm{C} / \mathrm{O}$ ratios are typically small (Wilson et al. 2016) and that the accreted material lacks other volatile elements such as nitrogen and sulphur typical of cometary material (Xu et al. 2017).
}

the oxygen-excess mass can be matched to the present-day hydrogen abundance if accretion occurred $\simeq 4 \mathrm{Myr}$ ago, implying around 60 percent of the parent body mass to be composed of water-ice, i.e. a $1.7 \times 10^{25}$ g overall parent-body mass $(\sim 0.2$ lunar masses $)$. For comparison, the dwarf planet Ceres is estimated to have a water-ice mass-fraction between 17 and 25 percent (McCord \& Sotin 2005). Of the remaining mass around $5 \times 10^{24} \mathrm{~g}$ would be composed of $\mathrm{MgO}$ and $\mathrm{SiO}_{2}$, and around $1 \times 10^{24} \mathrm{~g}$ composed of $\mathrm{FeO}$.

The above argument cannot be made assuming the GTC oxygen abundance: while increasing the look-back time increases the overall mass of accreted oxygen, as previously discussed the oxygen-excess is erased at about $5 \mathrm{Myr}$ leaving no oxygen that could be associated with the trace hydrogen (the absolute oxygen excess peaks about one order of magnitude less than required to fully correspond to the hydrogen abundance). However, if the planetesimal was accreted more recently, e.g. 2 Myr ago, a substantial oxygen excess remains $(\simeq 50$ percent at $2 \mathrm{Myr}$ ), still necessitating water-ice to be a major component of the parent body. In this case however, only some of the hydrogen in the atmosphere of SDSS J0956+5912 can be associated with the present metal abundances, with the remaining hydrogen having been accumulated from previous episodes of water-rich accretion.

\subsection{Accretion of core material as an unlikely scenario}

Recently Harrison et al. (2021) reanalysed most of the Hollands et al. (2017) sample. While recognising that SDSS J0956+5912 shows Mgenhanced abundances at present, their analysis led to the conclusion that this star accreted core-like material of which most of the Fe and $\mathrm{Ni}$ has diffused out of the atmosphere. We show that this scenario is unlikely. We first consider the composition of accreted planetary cores which can be expected to only show traces of $\mathrm{Mg}$. In Hollands et al. $(2017,2018)$, the white dwarf SDSS J0823+0546 is among the most-core like with abundances dominated by $\mathrm{Fe}$ and $\mathrm{Ni}$, and $\log (\mathrm{Mg} / \mathrm{Fe})=-1.15$. For SDSS J0956+5912, we instead find $\log (\mathrm{Mg} / \mathrm{Fe})=+1.4$. From Table 4 the diffusion timescale for $\mathrm{Mg}$ is around twice that for $\mathrm{Fe}$, and so given enough time the $\mathrm{Mg} / \mathrm{Fe}$ ratio could switch to $\mathrm{Mg}$-dominated. Using equation 3 from Hollands et al. (2018), it can be demonstrated that for $\mathrm{Mg} / \mathrm{Fe}$ to increase by 2.55 dex requires changes of $\Delta \log (\mathrm{Mg} / \mathrm{He})=-2.75 \mathrm{dex}$ and $\Delta \log (\mathrm{Fe} / \mathrm{He})=-5.30$ dex over a period of $11 \mathrm{Myr}$. Ignoring changes in the convection zone size due to the extreme implied metal abundances, the parent-body itself would have a mass of $\simeq 3 \times 10^{27} \mathrm{~g}$, i.e. about half the mass of the Earth (Figure 6). Given that planetary systems contain only a few objects with masses $>10^{27} \mathrm{~g}$ we consider this scenario extremely unlikely in comparison to accretion of a body of $\sim 10^{25} \mathrm{~g}$, for which far more bodies may be available and can be more easily scattered towards the white dwarf.

In summary, the material accreted by SDSS J0956+5912 resembles water ice mixed with magnesium silicates under the assumption of accretion in the last $4 \mathrm{Myr}$. While a less extreme composition may be possible assuming accretion occurred beyond $10 \mathrm{Myr}$ ago, such a scenario is a-priori unlikely due to the required parent-body mass exponentially increasing with time post-accretion, which is in tension with a mass-distribution of exoplanetesimals rapidly decreasing towards higher masses.

\section{CONCLUSIONS}

In general we find that while NUV spectroscopy provides additional constraints on DZ white dwarf atmospheric parameters, optical spectra - which can be obtained from ground based observatories - are 
sufficient for an accurate analysis in most cases. However, in the case of SDSS J0956+5912, the addition of NUV spectra resulting in a larger discrepancy, indicating that additional care should be taken when modelling spectra of warm, highly metal-rich DZs when only optical spectra are available. For SDSS J0956+5912 we measured extreme abundances of oxygen, magnesium, and silicon; indicating the accreted parent body was likely composed of $\mathrm{MgO}, \mathrm{SiO}_{2}$, and water-ice, with a total parent-body mass of up to $\simeq 0.2$ lunar masses (for accretion having occurred in the last $4 \mathrm{Myr}$ ). Furthermore we tentatively detect carbon at SDSS J0956+5912 suggesting it may have had a spectral type DQ before accreting its present abundances, but we demonstrate that this cannot explain the lack of observed of DZQs/DQZs more generally.

\section{ACKNOWLEDGEMENTS}

The authors acknowledge the referee, P. Dufour, who provided useful comments that improved the quality of this manuscript. This research received funding from the European Research Council under the European Union's Horizon 2020 research and innovation programme number 677706 (WD3D). BTG was supported by a Leverhulme Research Fellowship and the UK STFC grant ST/T000406/1. Based on observations made with the Gran Telescopio Canarias (GTC), installed in the Spanish Observatorio del Roque de los Muchachos of the Instituto de Astrofísica de Canarias (IAC), in the island of La Palma, and on observations made with the William Herschel Telescope (WHT) which is operated on the island of La Palma by the Isaac Newton Group of Telescopes in the Spanish Observatorio del Roque de los Muchachos of the IAC, and on observations made with the NASA/ESA Hubble Space Telescope, obtained at the Space Telescope Science Institute, which is operated by the Association of Universities for Research in Astronomy, Inc., under NASA contract NAS 5-26555. These observations are associated with programmes \#14128. Data for this paper have been obtained under the International Time Programme of the CCI (International Scientific Committee of the Observatorios de Canarias of the IAC).

\section{DATA AVAILABILITY}

All spectroscopic data used in this work were either obtained from public databases (SDSS), or are now publicly available from the respective observatory archives having exceeded their proprietary periods. For all $H S T$ data, the programme number is 14128 . WHT ISIS spectra can be found from the CASU archive on nights 20131228 and 20131229. GTC spectra can be found from the GTC archive with programme number GTC1-16ITP.

\section{REFERENCES}

Allard N. F., Leininger T., Gadéa F. X., Brousseau-Couture V., Dufour P., 2016a, A\&A, 588, A142

Allard N. F., Guillon G., Alekseev V. A., Kielkopf J. F., 2016b, A\&A, 593, A13

Bédard A., Bergeron P., Brassard P., Fontaine G., 2020, arXiv e-prints, p. arXiv:2008.07469

Blouin S., 2020, MNRAS,

Blouin S., Dufour P., 2019, MNRAS, 490, 4166

Blouin S., Dufour P., Allard N. F., 2018, ApJ, 863, 184

Blouin S., Dufour P., Allard N. F., Salim S., Rich R. M., Koopmans L. V. E., 2019a, ApJ, 872, 188
Blouin S., Allard N. F., Leininger T., Gadéa F. X., Dufour P., 2019b, ApJ, 875,137

Coutu S., Dufour P., Bergeron P., Blouin S., Loranger E., Allard N. F., Dunlap B. H., 2019, ApJ, 885, 74

Cunningham T., et al., 2021, MNRAS, 503, 1646

Cunto W., Mendoza C., Ochsenbein F., Zeippen C. J., 1993, A\&A, 275, L5

Debes J. H., Sigurdsson S., 2002, ApJ, 572, 556

Duncan M. J., Lissauer J. J., 1998, Icarus, 134, 303

Farihi J., Gänsicke B. T., Steele P. R., Girven J., Burleigh M. R., Breedt E., Koester D., 2012, MNRAS, 421, 1635

Farihi J., Gänsicke B. T., Koester D., 2013, Science, 342, 218

Gänsicke B. T., Marsh T. R., Southworth J., Rebassa-Mansergas A., 2006, Science, 314, 1908

Gänsicke B. T., Koester D., Farihi J., Girven J., Parsons S. G., Breedt E., 2012, MNRAS, 424, 333

Gänsicke B. T., Schreiber M. R., Toloza O., Fusillo N. P. G., Koester D., Manser C. J., 2019, Nat, 576, 61

Gentile Fusillo N. P., Gänsicke B. T., Farihi J., Koester D., Schreiber M. R., Pala A. F., 2017, MNRAS, 468, 971

Gentile Fusillo N. P., et al., 2021, MNRAS, 504, 2707

Girven J., Brinkworth C. S., Farihi J., Gänsicke B. T., Hoard D. W., Marsh T. R., Koester D., 2012, ApJ, 749, 154

Graham J. R., Matthews K., Neugebauer G., Soifer B. T., 1990, ApJ, 357, 216

Green G. M., et al., 2018, MNRAS, 478, 651

Green G. M., Schlafly E., Zucker C., Speagle J. S., Finkbeiner D., 2019, ApJ, 887,93

Guidry J. A., et al., 2021, ApJ, 912, 125

Harrison J. H. D., Bonsor A., Kama M., Buchan A. M., Blouin S., Koester D., 2021, MNRAS, 504, 2853

Hollands M. A., Koester D., Alekseev V., Herbert E. L., Gänsicke B. T., 2017, MNRAS, 467, 4970

Hollands M. A., Gänsicke B. T., Koester D., 2018, MNRAS, 477, 93

Hollands M. A., Tremblay P.-E., Gänsicke B. T., Koester D., Gentile-Fusillo N. P., 2021, Nat Astron., 5, 451

Horne K., 1986, PASP, 98, 609

Hoskin M. J., et al., 2020, MNRAS, 499, 171

Izquierdo P., Toloza O., Gänsicke B. T., Rodríguez-Gil P., Farihi J., Koester D., Guo J., Redfield S., 2021, MNRAS, 501, 4276

Jura M., 2003, ApJ Lett., 584, L91

Kaiser B. C., Clemens J. C., Blouin S., Dufour P., Hegedus R. J., Reding J. S., Bédard A., 2020, Sci, 370, abd1714

Klein B. L., Doyle A. E., Zuckerman B., Dufour P., Blouin S., Melis C., Weinberger A. J., Young E. D., 2021, ApJ, 914, 61

Koester D., 2010, Memorie della Societa Astronomica Italiana,, 81, 921

Koester D., Kepler S. O., 2019, A\&A, 628, A102

Koester D., Gänsicke B. T., Farihi J., 2014, A\&A, 566, A34

Koester D., Kepler S. O., Irwin A. W., 2020, A\&A, 635, A103

Kramida A., Ralchenko Y., Reader J., NIST ASD Team 2016, NIST Atomic Spectra Database (version 5.4), [Online], ht tp://physics.nist.gov/ asd

Manser C. J., et al., 2019, Sci, 364, 66

McCleery J., et al., 2020, MNRAS, 499, 1890

McCord T. B., Sotin C., 2005, J. Geophys. Res., 110, E05009

Melis C., et al., 2012, ApJ Lett., 751, L4

Mustill A. J., Veras D., Villaver E., 2014, MNRAS, 437, 1404

Paquette C., Pelletier C., Fontaine G., Michaud G., 1986, ApJS, 61, 197

Provencal J. L., Shipman H. L., Koester D., Wesemael F., Bergeron P., 2002, ApJ, 568, 324

Raddi R., Gänsicke B. T., Koester D., Farihi J., Hermes J. J., Scaringi S., Breedt E., Girven J., 2015, MNRAS, 450, 2083

Sackmann I.-J., Boothroyd A. I., Kraemer K. E., 1993, ApJ, 418, 457

Schatzman E., 1949, ApJ, 110, 261

Schröder K.-P., Connon Smith R., 2008, MNRAS, 386, 155

Swan A., Farihi J., Koester D., Holland s M., Parsons S., Cauley P. W., Redfield S., Gänsicke B. T., 2019, MNRAS, 490, 202

Turner S. G. D., Wyatt M. C., 2020, MNRAS, 491, 4672

Vanderbosch Z., et al., 2019, arXiv e-prints, p. arXiv:1908.09839

Vanderbosch Z. P., et al., 2021, arXiv e-prints, p. arXiv:2106.02659 
Vanderburg A., et al., 2015, Nat, 526, 546

Vanderburg A., et al., 2020, Nat, 585, 363

Veras D., Gänsicke B. T., 2015, MNRAS, 447, 1049

Veras D., Mustill A. J., Gänsicke B. T., Redfield S., Georgakarakos N., Bowler A. B., Lloyd M. J. S., 2016, MNRAS, 458, 3942

Villaver E., Livio M., 2007, ApJ, 661, 1192

Walkup R., Stewart B., Pritchard D. E., 1984, Phys. Rev. A, 29, 169

Wilson D. J., Gänsicke B. T., Koester D., Toloza O., Pala A. F., Breedt E., Parsons S. G., 2015, MNRAS, 451, 3237

Wilson D. J., Gänsicke B. T., Farihi J., Koester D., 2016, MNRAS, 459, 3282

Wolff B., Koester D., Liebert J., 2002, A\&A, 385, 995

Xu S., Zuckerman B., Dufour P., Young E. D., Klein B., Jura M., 2017, ApJ Lett., 836, L7

Zuckerman B., Becklin E. E., 1987, Nat, 330, 138

Zuckerman B., Koester D., Reid I. N., Hünsch M., 2003, ApJ, 596, 477

Zuckerman B., Koester D., Melis C., Hansen B. M., Jura M., 2007, ApJ, 671, 872

Zuckerman B., Melis C., Klein B., Koester D., Jura M., 2010, ApJ, 722, 725

Zuckerman B., Koester D., Dufour P., Melis C., Klein B., Jura M., 2011, ApJ, 739,101

This paper has been typeset from a $\mathrm{T}_{\mathrm{E}} \mathrm{X} / \mathrm{LT} \mathrm{E} \mathrm{X}$ file prepared by the author.

\begin{tabular}{|lll|}
$\mathrm{Na}_{2} \mathrm{O}$ & $\mathrm{SiO}_{2}$ & $\mathrm{FeO}$ \\
$\mathrm{MgO}$ & $\mathrm{CaO}$ & Excess \\
$\mathrm{Al}_{2} \mathrm{O}_{3}$ & & \\
\hline
\end{tabular}
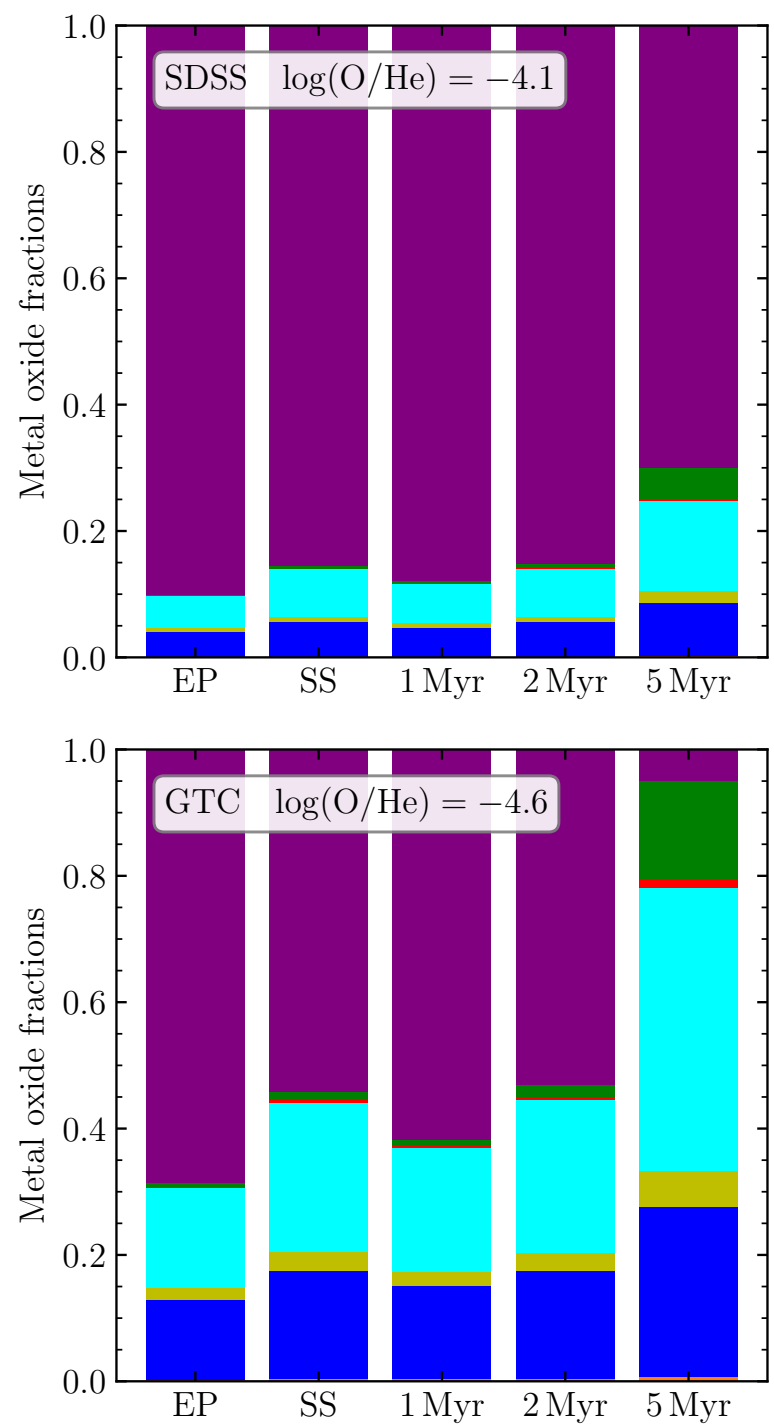

Figure 5. Metal oxide fractions and oxygen excess for the material accreted by SDSS J0956+5912 under different assumptions. The top and bottom panels reflect the different abundances measured for the SDSS and GTC spectra respectively. EP: Early phase accretion, where atmospheric abundances directly reflect the accreted composition. SS: In the steady-state phase accretion balances diffusion of metals out of the outer convection zone. 1-5 Myr: In the decreasing phase the observed a large fraction of the accreted metals may have already been depleted out of the outer convection zone, albeit at different rates. The three columns demonstrate the parent body composition assuming accretion occurring 1, 2 and $5 \mathrm{Myr}$ ago. 


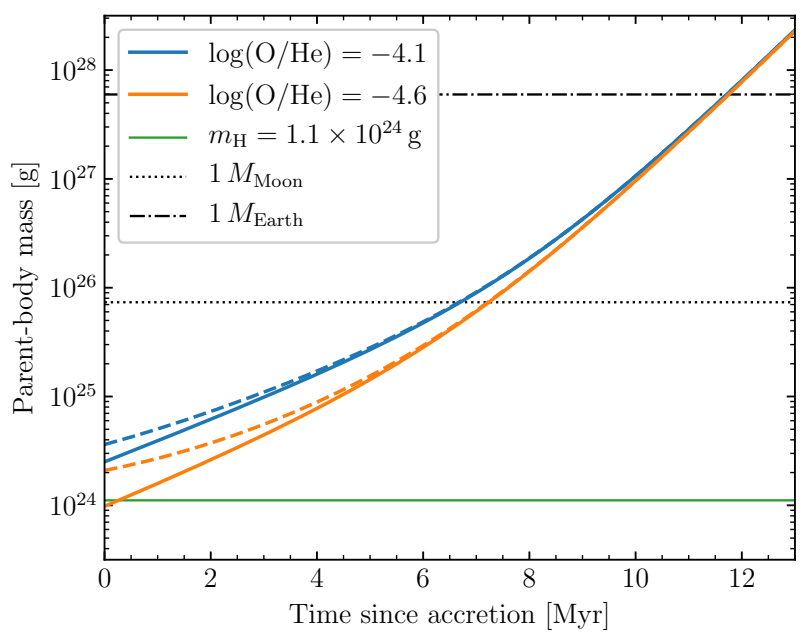

Figure 6. The inferred mass of the planetary object accreted by SDSS J0956+5912 as a function of how long ago accretion occurred. This is determined separately for both the SDSS and GTC oxygen abundances (blue and orange curves respectively). The parent-body mass when hydrogen is included in the calculations is shown by the dashed lines, and the hydrogen mass in the convection zone, $m_{\mathrm{H}}$, is shown by the solid green line. The curves converge for accretion times beyond $10 \mathrm{Myr}$ as oxygen becomes a relatively minor component of the total parent-body mass. All calculations assume a convection zone size and diffusion timescales corresponding to one-pressure scale height of convective-overshoot. 